\title{
MicroRNA-200a improves diabetic endothelial dysfunction by targeting KEAP1/NRF2
}

\author{
Ziping Jiang ${ }^{1}$ Junduo $\mathrm{Wu}^{2}$, Fuzhe $\mathrm{Ma}^{3}$, Jun Jiang ${ }^{4}$, Linlin $\mathrm{Xu}^{5}$, Lei $\mathrm{Du}^{6}$, Wenlin Huang7, Zhaohui Wang8, Ye Jia9?, \\ Laijin Lu' ${ }^{1}$ and Hao Wu6 \\ 1Department of Hand and Foot Surgery, The First Hospital of Jilin University, Changchun, Jilin, China \\ 2Department of Cardiology, The Second Hospital of Jilin University, Changchun, Jilin, China \\ ${ }^{3}$ Department of Nephrology, The First Hospital of Jilin University, Changchun, Jilin, China \\ 4Department of Neurosurgery, The Second Hospital of Shandong University, Jinan, Shandong, China \\ 5Department of Neurology, The Second Hospital of Shandong University, Jinan, Shandong, China \\ ${ }^{6}$ Department of Nutrition and Food Hygiene, School of Public Health, Shandong University, Jinan, Shandong, China \\ 7School of Science and Technology, Georgia Gwinnett College, Lawrenceville, Georgia, USA \\ ${ }^{8}$ Department of Acupuncture and Tuina, Changchun University of Chinese Medicine, Changchun, Jilin, China \\ 9Department of Diabetes Complications and Metabolism, Diabetes Metabolism Research Institute, Beckman Research Institute of City of Hope, \\ Duarte, California, USA
}

Correspondence should be addressed to L Lu or H Wu: Ijlu@jlu.edu.cn or hwu@sdu.edu.cn

\begin{abstract}
Over a half of the diabetic individuals develop macrovascular complications that cause high mortality. Oxidative stress (OS) promotes endothelial dysfunction (ED) which is a critical early step toward diabetic macrovascular complications. Nuclear factor erythroid 2-related factor 2 (NRF2) is a master regulator of cellular antioxidant defense system and combats diabetes-induced OS. Previously, we found that impaired NRF2 antioxidant signaling contributed to diabetes-induced endothelial OS and dysfunction in mice. The and diabetic ED. In aortic endothelial cells (ECs) isolated from C57BL/6 wild-type (WT) mice, high glucose (HG) reduced miR-200a levels and increased the expression of kelch-like ECH-associated protein 1 (Keap1) - a target of miR-200a and a negative regulator of NRF2. This led to the inactivation of NRF2 signaling and exacerbation of OS and inflammation. miR-200a mimic (miR-200a-M) or inhibitor modulated KEAP1/NRF2 antioxidant signaling and manipulated OS and inflammation under HG conditions. These effects were completely abolished by knockdown of Keap1, indicating that Keap1 mRNA is a major target of miR-200a. Moreover, the protective effect of miR-200a-M was completely abrogated in aortic ECs isolated from C57BL/6 Nrf2 knockout (KO) mice, demonstrating that NRF2 is required for miR-200a's actions. In vivo, miR-200a-M inhibited aortic Keap1 expression, activated NRF2 signaling, and attenuated hyperglycemia-induced OS, inflammation and ED in the WT, but not Nrf2 KO, mice. Therefore, the present study has uncovered miR-200a/KEAP1/NRF2 signaling that controls aortic endothelial antioxidant capacity, which protects against diabetic ED.
\end{abstract}
Key Words
- aorta
- diabetes
- endothelial dysfunction
- miR-200a
- NRF2 


\section{Introduction}

Macrovascular diseases develop in over a half of the diabetic individuals, posing a severe threat to public health (Forbes et al. 2004). Endothelial dysfunction (ED) is a critical early event in the pathogenesis of diabetic vascular complications (Sena et al. 2013, Sharma et al. 2017). Thus, it is essential to improve ED, the benefit from which may prevent or slow-down the progression of diabetic macrovascular complications.

Evidence has shown that hyperglycemia promotes oxidative stress (OS) (Giacco \& Brownlee 2010) which contributes to ED (Ceriello et al. 2013). Under hyperglycemia, an aberrant production of reactive oxygen species (ROS) exceeds the scavenging capacity of the antioxidant defense system, enhancing OS (Betteridge 2000, Incalza et al. 2018). This has brought to light the activation of the antioxidant defense system as a viable strategy to improve diabetic ED (Laight et al. 2000).

Nuclear factor erythroid 2-related factor 2 (NRF2) is a master regulator of the cellular antioxidant defense system (Kaspar et al. 2009, Zheng et al. 2011). In the nucleus, NRF2 activates the transcription of a series of genes, such as $\mathrm{NAD}(\mathrm{P}) \mathrm{H}$ dehydrogenase quinone 1 (Nqo1) and heme oxygenase 1 (Ho1) (Ruiz et al. 2013), the protein products of which function as antioxidants that scavenge upon excessive diabetes mellitus (DM)-generated free radicals. In the cytoplasm, NRF2 is sequestered by kelch-like ECH-associated protein 1 (KEAP1) which restricts NRF2 from nuclear translocation and facilitates proteasomal degradation of NRF2 (Zhang \& Hannink 2003). Thus, inhibition of KEAP1 may offer protection against diabetic ED by activation of the NRF2 antioxidant signaling.

In recent years, NRF2 activation has been achieved by small molecule-induced structural inactivation of KEAP1 protein function. However, little attention has been paid to inhibition of Keap1 gene expression, which may be an effective strategy to activate NRF2. Notably, inhibition of Keap1 gene expression has not been previously studied for its effect on diabetic ED, encouraging the investigation of this effect in the present study.

MicroRNAs (miRNAs) are short non-coding RNAs that act as suppressors of protein expression by inducing translational arrest or degradation of mRNAs, affecting almost every key cellular function (Fabian et al. 2010). MicroRNA-200a (miR-200a) was primarily reported to target and degrade Keap1 mRNA in breast cancer (Eades et al. 2011). We and others have found that miR-200a is upstream of KEAP1/NRF2 signaling in animal models of diabetic nephropathy (Wei et al. 2014, Wu et al. 2016).
In particular, specific inhibition of miR-200a increased renal Keap1 mRNA and protein expression, which in turn blunted renal NRF2 antioxidant signaling and exacerbated diabetic nephropathy in mice (Wei et al. 2014, Wu et al. 2016). These findings urge the investigation of the expression and action of miR-200a/KEAP1/NRF2 signaling in the diabetic endothelium, as well as the impact of miR-200a supplementation on ED.

In the present study, we first explored the effect of high glucose (HG) on aortic endothelial cells (ECs), showing that HG altered miR-200a/KEAP1/NRF2 signaling, and enhanced OS and inflammation in a time-dependent manner. Moreover, the role of miR-200a in controlling the expression and function of the KEAP1/NRF2 signaling in ECs was confirmed by using specific mimic and inhibitor of miR-200a. As miR-200a may target multiple mRNAs, the role of KEAP1 in mediating miR-200a's actions in ECs was explored by using a siRNA (siRNA) for Keap1 mRNA, in the presence of miR-200a mimic (miR-200a-M). Further, by using ECs isolated from C57BL/6 wild-type (WT) and Nrf2 gene-knockout (KO) mice, NRF2 was investigated for its requirement for miR-200a's effects on HG-induced OS and inflammation. Finally, to assess the role of miR-200a/KEAP1/NRF2 signaling in diabetic ED, WT and $\mathrm{Nrf2}$ KO diabetic mice were treated with miR-200a-M.

\section{Materials and methods}

\section{Cell culture and experiments}

ECs were isolated from the aortas of 8-week-old C57BL/6 WT or Nrf2 KO male mice and were cultured under normal glucose (NG, $1 \mathrm{~g} / \mathrm{L}$ ) conditions, as described in our previous studies (Wu et al. 2018a,b).

To observe the time-dependent impact of $\mathrm{HG}$ on the expression of miR-200a/KEAP1/NRF2 signaling, OS and inflammation, ECs isolated from the WT mice (WT ECs) were exposed to HG $(4.5 \mathrm{~g} / \mathrm{L})$ or mannitol $(3.64 \mathrm{~g} / \mathrm{L})$, for $1,6,12,24$ and $48 \mathrm{~h}$. To study the role of miR-200a in controlling KEAP1/NRF2, OS and inflammation under $\mathrm{HG}$ conditions, WT ECs were challenged by HG with miR-200a-M (30 nM, Thermo Fisher Scientific) or a specific inhibitor of miR-200a (miR-200a-I, $30 \mathrm{nM}$, Thermo Fisher Scientific), for $48 \mathrm{~h}$. To verify the role of KEAP1 in mediating miR-200a's action, WT ECs were co-treated with HG and miR-200a-M, with Keap1 silenced using an siRNA (100 nM, GenePharma, Suzhou, China), for $48 \mathrm{~h}$. The Keap1-siRNA was introduced into ECs using the transfection reagent 
Lipofectamine $^{\circledR} 2000$ (Thermo Fisher Scientific). To investigate the requirement of NRF2 in miR-200a's action, ECs isolated from the Nrf2 $\mathrm{KO}$ mice (KO ECs) were treated with HG and miR-200a-M (30 nM), for $48 \mathrm{~h}$. The doses of miR-200a-M, miR-200a-I and Keap1-siRNA used were based on our preliminary work and were similar to those previously reported (Kim et al. 2012, Wang et al. 2014, Barwari et al. 2018).

\section{Analysis of ROS and lipid peroxides}

Cellular ROS and malondialdehyde (MDA) levels were measured using assay kits from Sigma-Aldrich, following the manufacturer's instructions.

\section{Analysis of nitric oxide production}

In order to evaluate endothelial function, the generation of NO was measured using 3-amino,4-aminomethyl-2', 7'-difluorescein diacetate (DAF-FM DA, Sigma-Aldrich) as previously described (Yang et al. 2015).

\section{Animal housing and experiments}

C57BL/6 WT (Nrf2+/+) and Nrf2 KO (Nrf2-/-) mice were obtained through breeding of heterozygotes (Nrf2+/-) (Zheng et al. 2011, Sun et al. 2017). The mice were housed in the Animal Center of Jilin University at $22^{\circ} \mathrm{C}$, on a $12: 12$-h light-dark cycle, with free access to rodent feed and tap water. The Institutional Animal Care and Use Committee at Jilin University approved all the experimental procedures which meets the UK Animals (Scientific Procedures) Act, 1986 and associated guidelines.

Eight-week-old male WT and Nrf2 $\mathrm{KO}$ mice were injected intraperitoneally with sodium citrate or streptozotocin (STZ, $50 \mathrm{mg} / \mathrm{kg} /$ day, dissolved in $0.1 \mathrm{M}$ sodium citrate, $\mathrm{pH} 4.5$; Sigma-Aldrich) once every day, for 5 consecutive days (Zheng et al. 2011, Kato et al. 2016, $\mathrm{Wu}$ et al. 2016). Fasting glucose levels (4-h fast) were determined 1 week after the last injection. Mice with fasting glucose levels above $13.89 \mathrm{mM}$ were considered diabetic. Blood glucose levels were recorded 4, 8, 12, 16 and 20 weeks post DM onset.

The WT and Nrf2 KO diabetic mice received s.c. injections of miR-200a-M or its negative control (both at $1 \mathrm{mg} / \mathrm{kg}, 3$ times weekly; Thermo Fisher) immediately after DM was confirmed, for a total period of 20 weeks. At the end of the procedures, the mice were killed under anesthesia by intraperitoneal injection of chloral hydrate
(0.3 $\mathrm{mg} / \mathrm{kg})$ (Pan et al. 2017), with the aortas harvested for analysis.

\section{Assessment of aortic ED}

To assess aortic ED, contractility in response to phenylephrine (PE) and relaxation in response to acetylcholine (ACh) were determined using thoracic aortas, as previously described (Peng et al. 2015, Wu et al. $2018 a$ ). The doses of PE and ACh were $10^{-9}, 10^{-8}, 10^{-7}, 10^{-6}$, $10^{-5}$ and $10^{-4} \mathrm{M}$.

\section{Analysis of aortic morphology}

The freshly harvested thoracic aortas were immediately fixed into $10 \%$ buffered formalin solution, embedded in paraffin, and sectioned into 5 - $\mu \mathrm{m}$-thick sections onto glass slides. Morphology of the aortas was evaluated using hematoxylin and eosin (H\&E) staining. Selection of areas to photograph was done by people blind to the identity of the samples.

\section{Immunohistochemical (IHC) staining}

IHC staining was performed as previously described (Wu et al. 2015), using primary antibodies against KEAP1 (1:100; Santa Cruz Biotechnology), NRF2 (1:100; Santa Cruz Biotechnology) and 4-hydroxynonenal (4-HNE, 1:100; Alpha Diagnostic Int., San Antonio, TX, USA). Positive area of 4-HNE was quantified within the full-thickness of the artery wall. Selection of areas to photograph and scoring was done by people blind to the identity of the samples.

\section{Quantitative real-time PCR (qPCR)}

qPCR was performed using aortic tissue and lysate of ECs as previously described (Deshpande et al. 2013, Wu et al. 2015). For miR-200a, U6 was used as an endogenous control, and Gapdh for the others. The primers for Gapdh, Ho1, inducible nitric oxide synthase (iNos), Keap1, miR-200a, monocyte chemoattractant protein 1 (Mcp-1), Nqo1, U6 and Vcam-1 were provided by Thermo Fisher Scientific.

\section{Western blot}

Western blot analysis was performed as previously described (Wu et al. 2015, 2018a, Yaribeygi et al. 2018), 
using cell lysates and aortic tissue, with primary antibodies against GAPDH (1:3000; Santa Cruz Biotechnology), KEAP1 (1:1000; Santa Cruz Biotechnology), NRF2 (1:1000, Santa Cruz Biotechnology) and histone H3 (1:1000; Santa Cruz Biotechnology). Histone H3 was used as an endogenous control for nuclear NRF2 (n-NRF2), and GAPDH for the others.

\section{Isolation of nuclei}

In order to determine the level of n-NRF2, nuclei were isolated from ECs using a nuclear and cytoplasmic extraction kit from Thermo Fisher Scientific, following the manufacturer's instructions.

\section{Statistical analysis}

Cell experiments were performed in triplicate. Seven mice per group were studied. Western blot images were analyzed using Image Studio Lite (LI-COR Biosciences, Lincoln, NE, USA). IHC-positive area was quantified using Image Pro Plus 6.0 software (Media Cybernetics, Rockville, MD, USA). One-way ANOVA was performed for the comparisons among the WT or KO groups. Twoway ANOVA was used for the comparisons among the WT and $\mathrm{KO}$ groups. The measurements for each group were summarized as means \pm s.D. A test is significant if $P<0.05$.

\section{Results}

\section{HG altered endothelial miR-200a/KEAP1/NRF2 signaling, enhancing OS and inflammation in a time-dependent manner}

To investigate the expression of miR-200a, Keap1 and NRF2 antioxidant signaling, and the levels of OS and inflammation in response to a time series of HG incubation, ECs were exposed to HG for $1 \mathrm{~h}, 6 \mathrm{~h}, 12 \mathrm{~h}, 24 \mathrm{~h}$ and $48 \mathrm{~h}$. HG time-dependently decreased miR-200a levels (Fig. 1A), and enhanced Keap1 mRNA (Fig. 1B) and protein (Fig. 1C) expression. This decreased whole cellular NRF2 (total NRF2, t-NRF2) at $24 \mathrm{~h}$ and $48 \mathrm{~h}$ (Fig. 1D). Different from the pattern of t-NRF2 expression, HG incubation increased n-NRF2 at $6 \mathrm{~h}$ and $12 \mathrm{~h}$, but decreased n-NRF2 thereafter, ending with a drastic decrease in n-NRF2 level at $48 \mathrm{~h}$ (Fig. 1E). The mRNA levels of the NRF2 downstream antioxidant genes $\mathrm{Nqo1}$ and $\mathrm{Ho} 1$ were elevated by HG at $12 \mathrm{~h}$ and $24 \mathrm{~h}$ and were downregulated by HG at $48 \mathrm{~h}$. The levels of ROS and MDA, and the mRNA levels of
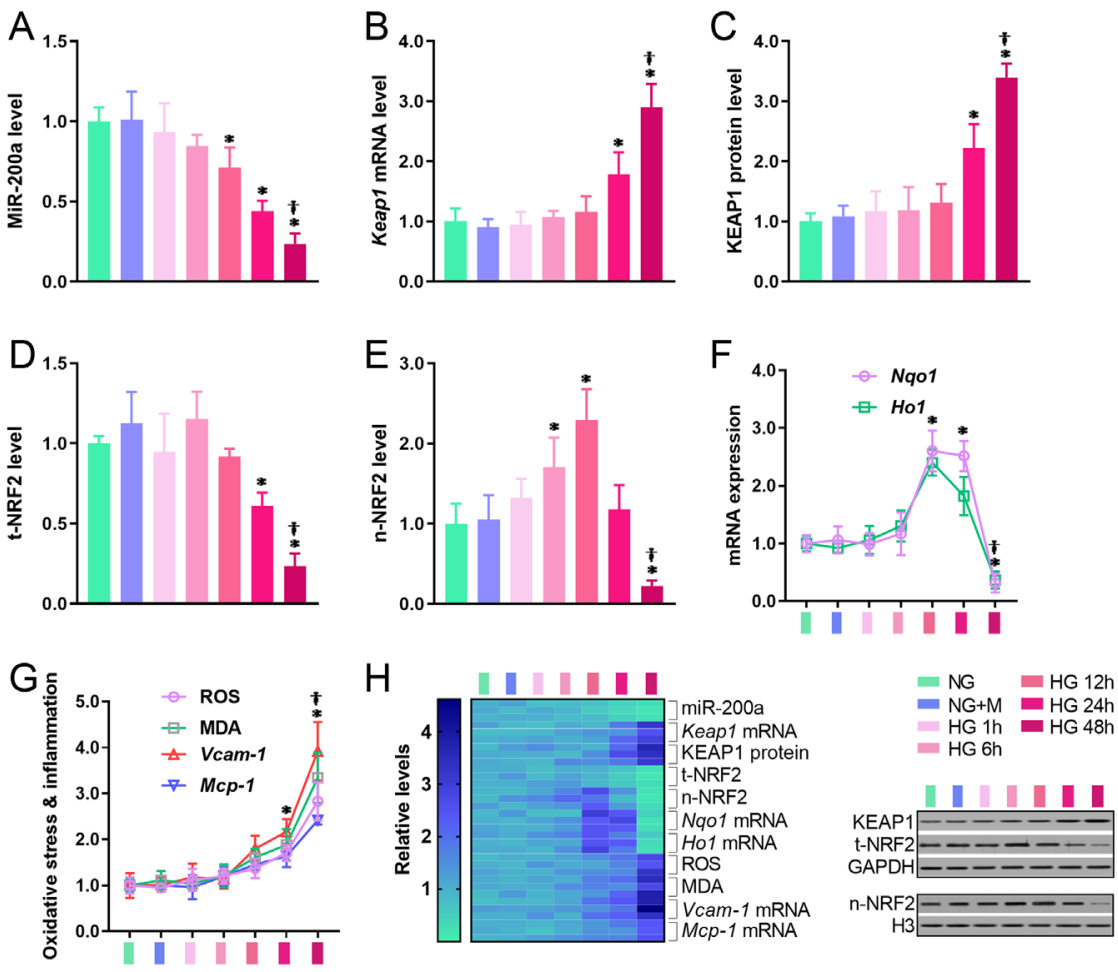

\section{Figure 1}

HG altered endothelial miR-200a/KEAP1/NRF2 signaling, enhancing OS and inflammation in a time-dependent manner. ECs isolated from C57BL/6 WT mice were cultured under NG conditions and were treated with mannitol or $\mathrm{HG}$ for $1 \mathrm{~h}, 6 \mathrm{~h}, 12 \mathrm{~h}, 24 \mathrm{~h}$ and $48 \mathrm{~h}$. The levels of (A) miR-200a, Keap1 (B) mRNA and (C) protein, (D) t-NRF2, (E) n-NRF2, and (F) the mRNA expression of $\mathrm{NqO} 1$ and $\mathrm{Ho} 1$ were determined by qPCR or Western blot. (G) The mRNA levels of the pro-inflammatory genes Vcam-1 and Mcp-1 were measured by qPCR. (G) ROS and MDA levels were determined using commercial kits to assess OS. $(H)$ The relative levels of these indices were summarized as a heatmap. The data was normalized to the NG group and presented as means \pm s.D. $(n=3) . * P<0.05$ vs NG. ${ }^{\dagger} P<0.05$ vs HG 24 h. H3, histone H3; HG, high glucose; Ho1, heme oxygenase 1; Keap1, kelch-like ECHassociated protein 1; M, mannitol; miR-200a, microRNA-200a; Mcp-1, monocyte chemoattractant protein 1; MDA, malondialdehyde; NG, normal glucose; NRF2 nuclear factor erythroid 2-related factor 2; n-NRF2, nuclear NRF2; Nq01, NAD(P)H dehydrogenase quinone 1 ; OS, oxidative stress; qPCR, quantitative real-time PCR; ROS, reactive oxygen species; t-NRF2, total/whole cellular NRF2; Vcam-1, vascular cell adhesion molecule-1; WT, wild-type. 
Vcam-1 and Mcp-1 were increased by HG incubation for 24 and 48 h (Fig. 1G). Forty-eight-hour HG incubation dramatically enhanced OS and inflammation (Fig. 1G). This coincided with the HG-enhanced Keap1 gene expression (Fig. 1B and C) and the HG-induced inhibition of miR-200a and NRF2 antioxidant system at $48 \mathrm{~h}$ (Fig. $1 \mathrm{D}, \mathrm{E}$ and F), suggesting opposing effects of miR-200a and KEAP1 in the control of NRF2 antioxidant signaling, and in the modulation of OS and inflammation under $\mathrm{HG}$ conditions (Fig. 1H).

\section{miR-200a controlled the KEAP1/NRF2 antioxidant system, combating HG-induced OS and inflammation}

We aimed to verify whether miR-200a controls the endothelial KEAP1/NRF2 antioxidant system and whether miR-200a is capable of diminishing oxidative and inflammation in ECs under 48-h incubation with HG. miR-200a-M supplementation increased miR-200a levels (Fig. 2A) and blunted the HG-induced expression of Keap1 (Fig. 2B and C). This elevated the levels of t-NRF2 (Fig. 2D), n-NRF2 (Fig. 2E), and the mRNA expression of Nqo1 and Ho1 (Fig. 2F), alleviating the HG-induced OS and inflammation (Fig. 2G). On the contrary, inhibition of miR-200a by miR-200a-I (Fig. 2A) further increased Keap1 mRNA and protein levels compared with NC-I under HG conditions (Fig. 2B and C), resulting in a further decrease in the levels of t-NRF2 (Fig. 2D), n-NRF2 (Fig. 2E), and mRNA levels of Nqo1 and Ho1 (Fig. 2F). Moreover, miR-200a-I exacerbated the HG-induced endothelial OS and inflammation (Fig. 2G). These results demonstrate that alteration of miR-200a levels remarkably changed the expression of KEAP1/NRF2 antioxidant system and the status of OS and inflammation under HG conditions, suggesting that miR-200a governs endothelial antioxidant activity that combats endothelial OS and inflammation.

\section{KEAP1 predominantly mediated miR-200a's actions in ECs under HG conditions}

Because miR-200a may target several mRNAs that might contribute to miR-200a's actions upon HG-induced endothelial injury, we investigated the role of KEAP1 in mediating miR-200a's actions in HG-treated ECs by using Keap1-siRNA, in the presence of miR-200a-M. The Keap1siRNA effectively reduced Keap1 mRNA and protein levels (Fig. 3A and B). miR-200a-M did not further decrease Keap1 expression (Fig. 3A and B) despite its remarkable elevation of miR-200a (Fig. 3C). In the presence of the Keap1-siRNA, miR-200a-M did not further enhance the expression of NRF2 antioxidant signaling (Fig. 3D, E and F), nor did it further inhibit OS and inflammation (Fig. 3G).
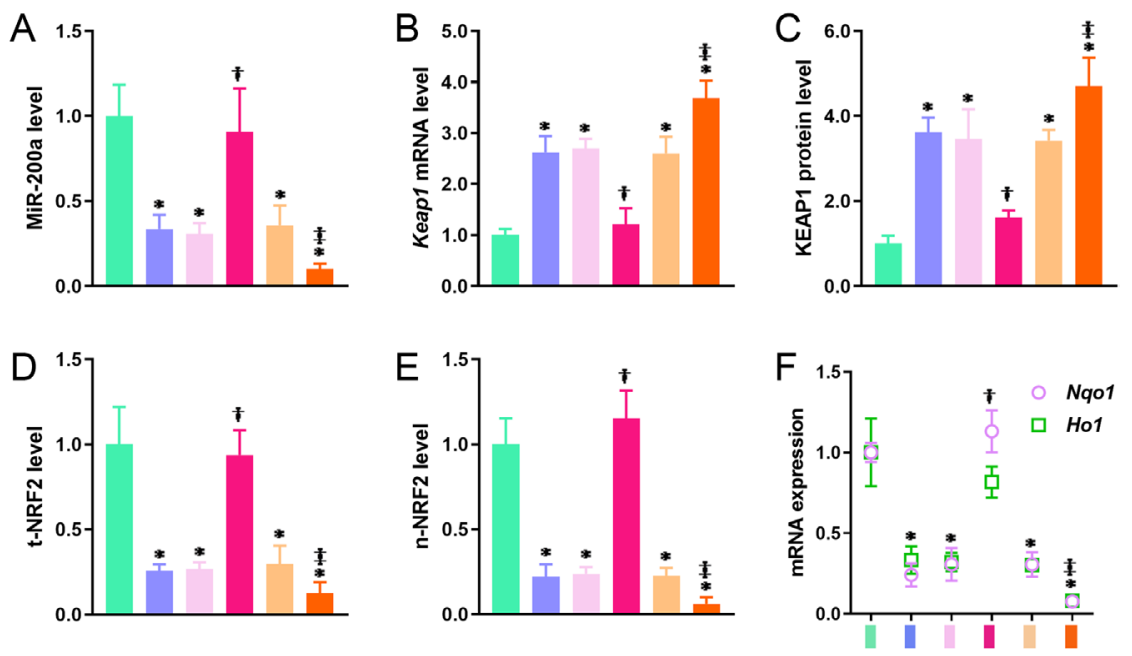
Figure 2
miR-200a controlled the KEAP1/NRF2 antioxidant system, combating HG-induced OS and inflammation. ECs isolated from WT mice were treated with miR-200a-M, miR-200a-l and their negative controls respectively, in the presence of HG for $48 \mathrm{~h}$. The expression of the miR-200a/ KEAP1/NRF2 signaling, including (A) miR-200a, Keap1 (B) mRNA and (C) protein, (D) t-NRF2, (E) n-NRF2, and (F) the mRNA levels of $\mathrm{NqO} 1$ and $\mathrm{Ho} 1$ were determined. Additionally, (G) ROS and MDA levels, and mRNA levels of Vcam-1 and Mcp-1 were measured to assess OS and inflammation. The data were normalized to the NG group and presented as means \pm S.D. $(n=3)$. ${ }^{*} P<0.05$ vs NG. ${ }^{\dagger} P<0.05$ vs HG + NC-M, ${ }^{\ddagger} P<0.05$ vs. HG + NC-I. miR-200a-I, miR-200a inhibitor; miR-200a-M, miR-200a mimic; NC-I, negative control of miR-200a-l; NC-M, negative control of miR-200a-M. Other abbreviations are the same as Fig. 1.

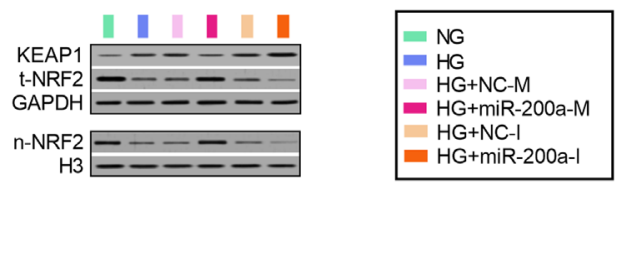



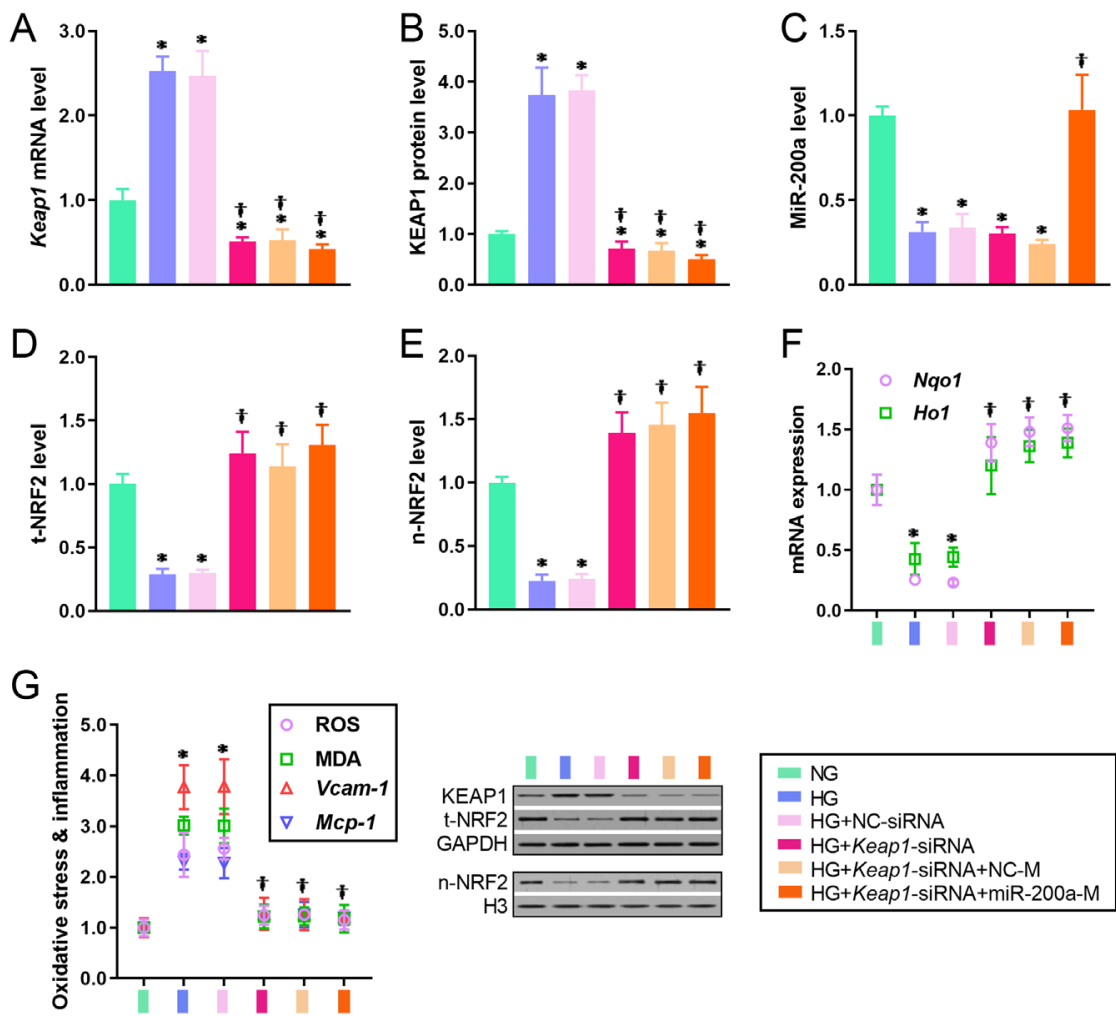

\section{Figure 3}

KEAP1 predominantly mediated miR-200a's actions in ECs under HG conditions. To investigate the role of KEAP1 in mediating miR-200a's efficacies, HG-treated ECs that were isolated from WT mice were co-treated with Keap1-siRNA, in the presence of miR-200a-M or NC-M, for $48 \mathrm{~h}$. The levels of Keap1 (A) mRNA and (B) protein, (C) miR-200a, (D) t-NRF2, (E) n-NRF2 were measured. The (F) mRNA levels of Nqo1 and Ho1, (G) levels of ROS and MDA, as well as mRNA levels of Vcam-1 and $M c p-1$ were determined. The data were normalized to the NG group and presented as means \pm s.D. $(n=3)$. ${ }^{\star} p<0.05$ vs NG. ${ }^{\dagger} P<0.05$ vs HG + NC-siRNA. NC-siRNA, negative control siRNA for Keap1; siRNA, small interfering RNA. Other abbreviations are the same as Figs 1 and 2 .
These findings indicate that KEAP1 is a critical target of miR-200a and predominantly mediates miR-200a's effects on endothelial antioxidant activity, OS and inflammation under HG conditions.

\section{NRF2 was required for miR-200a to attenuate HG-induced endothelial injury, and increase NO production}

To address whether or not NRF2 plays a role in miR-200a's actions upon HG, ECs isolated from WT and Nrf2 KO mice were subjected to $\mathrm{HG}$ for $48 \mathrm{~h}$, in the presence of miR-200a-M. miR-200a-M increased miR-200a levels (Fig. 4A), decreased Keap1 mRNA (Fig. 4B) and protein (Fig. 4C) levels in both the WT and Nrf2 KO ECs. NRF2 protein was undetectable in the Nrf2 KO ECs (Fig. 4D), confirming the deletion of the Nrf2 gene in these mice. In the absence of NRF2, miR-200a-M failed to elevate Nqo1 and Ho1 mRNA levels (Fig. 4E) and was unable to restrain OS and inflammation (Fig. 4F), the effects of which were in contrast to those in the WT ECs. HG generated less NO in the Nrf2 KO ECs compared with the WT ECs (Fig. 4G). Moreover, miR-200a supplementation was able to increase NO generation in the WT, but not Nrf2 KO, ECs. These findings demonstrate that miR-200a's anti-oxidative and anti-inflammatory activities are dependent on NRF2, and miR-200a drives endothelial NO production through activation of NRF2.

\section{miR-200a supplementation manipulated the aortic KEAP1/NRF2 antioxidant system under hyperglycemic conditions}

To further explore the effect of miR-200a supplementation on the aortic KEAP1/NRF2 antioxidant system under hyperglycemic conditions in the presence or absence of NRF2, STZ-induced WT and Nrf2 KO diabetic mice were treated with miR-200a-M for 20 weeks. The diabetic mice developed higher blood glucose levels compared with the non-diabetic control mice (Fig. 5A). miR-200a-M and its negative control did not have a significant impact on blood glucose levels (Fig. 5A). H\&E staining showed a mild derangement of smooth muscle cells and thickening of tunica media in both the WT and Nrf2 KO diabetic mice (Fig. 5B). Hyperglycemia resulted in reduction of miR-200a and elevation of Keap1 mRNA levels in the aortas of the WT and Nrf2 KO mice, the effects of which were reversed by miR-200a supplementation (Fig. 5C and D). Evident KEAP1 protein expression was observed in the aortas of the WT and Nrf2 KO diabetic mice, as shown by IHC staining (Fig. 5E) and Western blot (Fig. 5F). miR-200a-M decreased aortic KEAP1 protein in both WT 
A

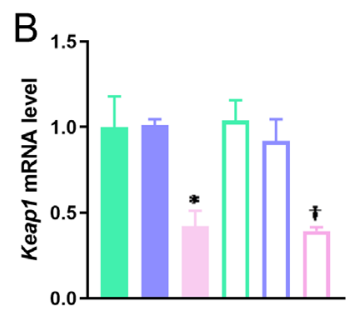

D

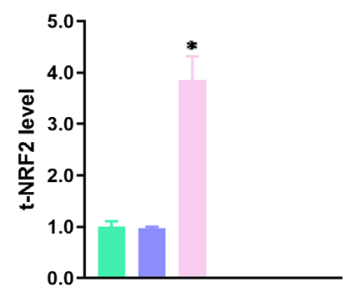

E



G

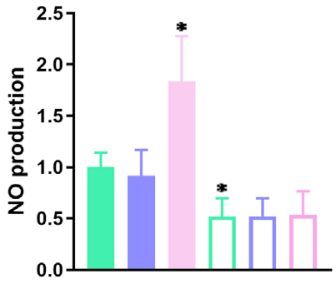

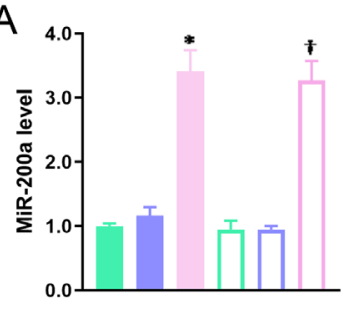
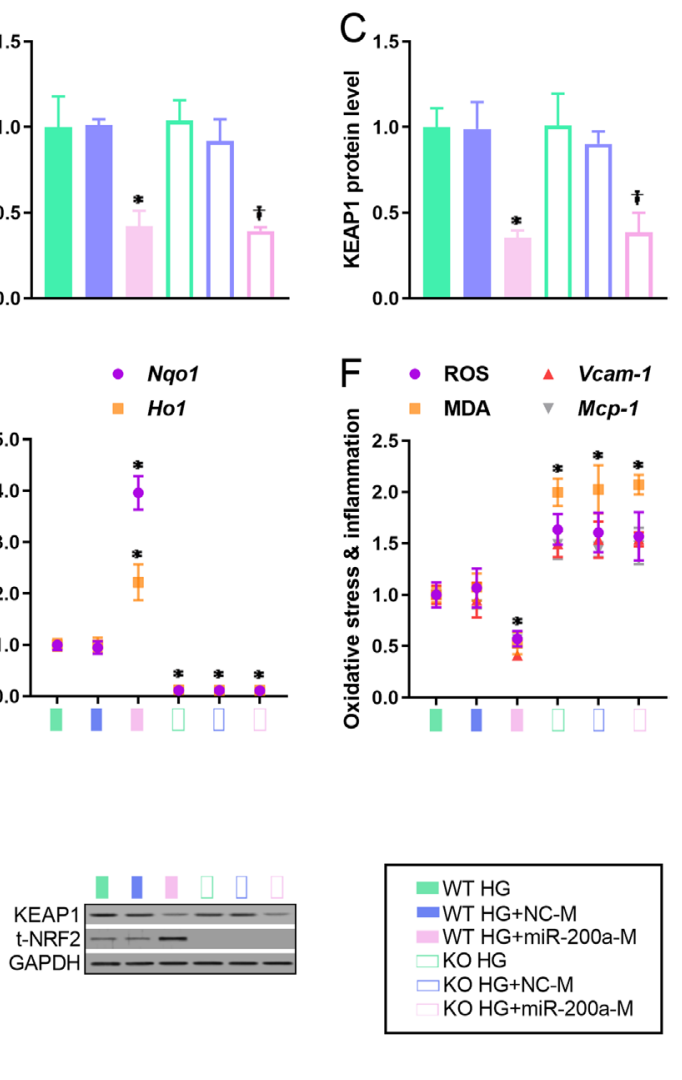

and Nrf2 KO diabetic mice (Fig. 5E and F). Moreover, miR-200a elevated NRF2 protein levels (Fig. 5G and H), and increased Nqo1 and Ho1 mRNA levels (Fig. 5I and J) in the aortas of the WT diabetic mice, the effects of which were completely abrogated in the Nrf2 KO diabetic mice (Fig. 5G, H, I and J).

\section{miR-200a-M improved hyperglycemia-induced aortic OS, inflammation and ED through activation of NRF2}

We investigated the effect of miR-200a-M on hyperglycemia-induced aortic OS, inflammation and ED. In WT diabetic mice, miR-200a-M reduced aortic iNos mRNA levels (Fig. 6A), positive stain of 4-HNE (Fig. 6B), and mRNA levels of Vcam-1 and Mcp-1 (Fig. 6C and D). Additionally, miR-200a-M alleviated the hyperglycemiaprovoked aortic contraction in response to $\mathrm{PE}$ at doses of $10^{-7}, 10^{-6}, 10^{-5}$ and $10^{-4} \mathrm{M}$ (Fig. 6E, left panel) and improved the hyperglycemia-impaired aortic relaxation in response to ACh at $10^{-7}, 10^{-6}$, and $10^{-4} \mathrm{M}$ in the WT mice (Fig. $6 \mathrm{~F}$, left panel). These effects of miR-200a-M were completely abolished in the Nrf2 KO diabetic mice (Fig. 6A, B, C and $\mathrm{D}$; right panels of $\mathrm{E}, \mathrm{F}$ ). These results demonstrate that miR-200a protects against hyperglycemia-induced aortic OS, inflammation and ED via activation of NRF2.

\section{Discussion}

\section{Figure 4}

NRF2 was required for miR-200a's capabilities to attenuate HG-induced endothelial injury and increase NO production. ECs isolated from either C57BL/6 WT or Nrf2 KO mice were challenged with $\mathrm{HG}$, in the presence of miR-200a-M or NC-M, for $48 \mathrm{~h}$. The expression of (A) miR-200a, (B) Keap1 mRNA, (C) KEAP1 protein, (D) t-NRF2, (E) Nqo1 mRNA and Ho1 mRNA were determined. OS and inflammation were assessed by measuring the levels of (F) ROS and MDA, as well as the mRNA levels of Vcam-1 and Mcp-1. (G) NO production was measured to assess endothelial function. The data were normalized to the WT HG group and presented as means \pm S.D. $(n=3) .{ }^{*} P<0.05$ vs WT $\mathrm{HG}+\mathrm{NC}-\mathrm{M} .{ }^{+} P<0.05$ vs KO HG + NC-M. KO, knockout; NO, nitric oxide. Other abbreviations are the same as Figs 1 and 2 .

As ED contributes to the pathogenesis of diabetic macrovascular complications, the present study has researched the role of miR-200a/KEAP1/NRF2 in the regulation of diabetic ED. The present study has found that (1) aortic endothelial miR-200a expression was suppressed under HG/hyperglycemic conditions, leading to overexpression of KEAP1 and inactivation of NRF2 antioxidant signaling; (2) inhibition and miR-200a supplementation modulated the expression and function of KEAP/NRF2 under HG conditions, and altered HG-induced endothelial OS and inflammation, suggesting that miR-200a is a key upstream regulator of KEAP1/NRF2 signaling that combats HG-induced endothelial injury; (3) by silencing Keap1 mRNA and using Nrf2 KO mice, KEAP1 and NRF2 were shown to predominantly mediate miR-200a's actions; (4) in vivo delivery of miR-200a attenuated hyperglycemia-induced aortic OS, inflammation and ED through activation of NRF2.

The most innovative finding of the present study is the identification of miR-200a in controlling hyperglycemiainduced ED. Mechanistically, this effect was through miR-200a-induced manipulation of the KEAP1/NRF2 antioxidant signaling, since miR-200a's actions were 
B

A

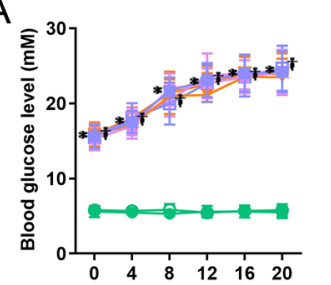

C

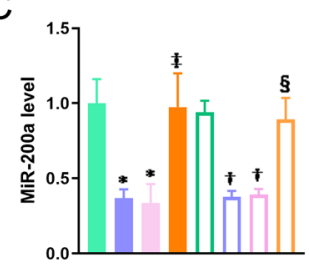

$\mathrm{F}$

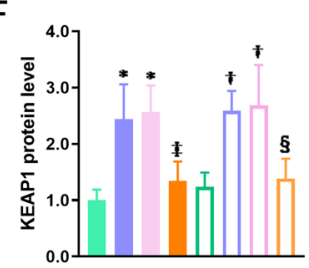

I

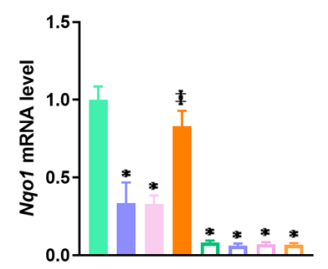

$\rightarrow$ WT Ctrl

- WT DM

\pm WT DM+NC-M

-WT DM+miR-200a-M

$\theta$ Ko Ctrl

$\because$ KO DM

$\triangle$ KO DM+NC-M

7 KO DM+miR-200a-M

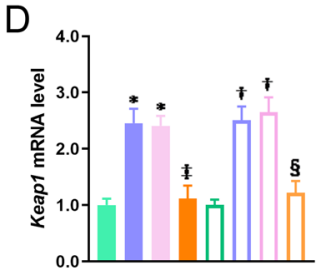

G

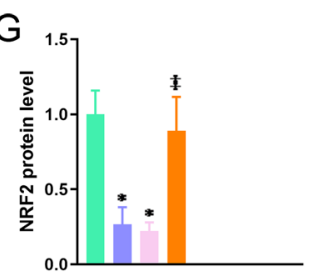

$\mathrm{J}$

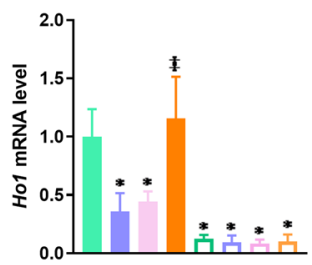

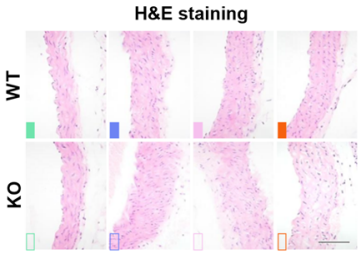

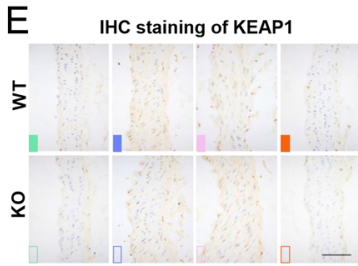

$\mathrm{H}$



(1) I I

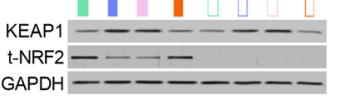

GAPDH

WT Ctrl

-WT DM

WT DM+NC-M

-WT DM+miR-200a-M

KO Ctrl

KO DM

KO DM+miR-200a-M

\section{Figure 5}

miR-200a supplementation manipulated the aortic KEAP1/NRF2 antioxidant system under hyperglycemic conditions. C57BL/6 WT and Nrf2 $\mathrm{KO}$ mice were induced to DM by streptozotocin and were treated with miR-200a-M or NC-M. (A) Blood glucose levels were recorded $0,4,8,12,16$, 20 weeks post DM onset. (B) H\&E staining was performed to assess aortic morphology. The expression of (C) miR-200a and (D) Keap1 mRNA were determined by qPCR. (E) Positive stain of KEAP1 protein in the aortas were detected by $\mathrm{IHC}$ staining. Protein levels of (F) KEAP1 and (G) NRF2 were determined by Western blot. $(\mathrm{H})$ Localization of NRF2 in the aortas was evaluated by IHC staining. mRNA levels of (I) Nqo1 and (I) Ho1 were measured by qPCR. For (C), (D), (F), (G), (I) and (J), the data was normalized to WT Ctrl. All the data were presented as means \pm s.D. $(n=7) . * P<0.05$ vs WT Ctrl. ${ }^{\ddagger} P<0.05$ vs KO Ctrl. ${ }^{\ddagger} P<0.05$ vs WT DM + NC-M. ${ }^{5}$ KO DM + NC-M. Ctrl, control; DM, diabetes mellitus; H\&E, hematoxylin and eosin; $\mathrm{IHC}$, immunohistochemical. Other abbreviations are the same as Figs 1, 2 and 4. almost completely abolished when Keap1 mRNA was knocked down or when the Nrf2 gene was deleted (Figs $3,4,5$ and 6). These findings demonstrate that the miR-200a/KEAP1/NRF2 pathway controls endothelial antioxidant activity under HG/hyperglycemic conditions and regulates endothelial function, providing miR-200a and Keap1 mRNA as novel targets for the intervention of diabetic macrovascular diseases. Previously, we and others have reported that renal miR-200a was decreased under diabetic conditions, resulting in increased Keap1 expression and blunted NRF2 antioxidant signaling. Despite these prior work on diabetic nephropathy, the role of miR-200a/KEAP1/NRF2 pathway in diabetesinduced endothelial dysfunction is unknown. Because endothelial dysfunction is a critical pathophysiological event prior to diabetic macrovascular complications, in the present study, we have characterized an important role of this pathway during diabetes-induced endothelial dysfunction. In support of our findings, a recent study showed that inhibition of histone deacetylase 3 prevented blood-brain barrier permeability in a mouse model of type $2 \mathrm{DM}$, at least in part attributed to miR-200a/KEAP1/NRF2 pathway (Zhao et al. 2019). However, different from Zhao's study, our work has demonstrated a central role of the miR-200a/KEAP1/NRF2 pathway in control of diabetes-induced aortic endothelial dysfunction, shedding light upon the intervention of diabetes-induced macrovascular diseases, such as atherosclerosis. Given the protective effects of miR-200a on multiple organs/tissues under diabetes, such as the aorta, kidney and brain, miR-200a supplementation might produce benefits in the prevention of several complications of diabetes. This should urge further study of this pathway in diabetes and its various complications.

NRF2 plays a crucial role in protection against diabetic ED. Previously, we found that Nrf2 gene deletion exacerbated DM-induced aortic endothelial OS, inflammation and dysfunction, demonstrating the requirement of NRF2 in self-protection against DM-induced endothelial injury (Wu et al. 2018b). In our previous (Wu et al. 2018b) and present work (Figs 1D, E, F and 5G, H, I, J), impaired NRF2 antioxidant signaling was 


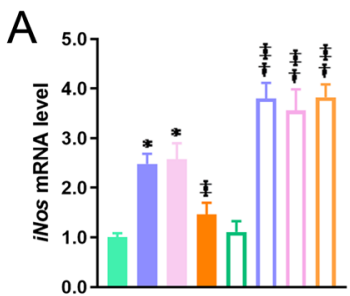

C

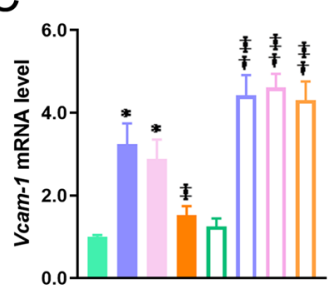

$E$

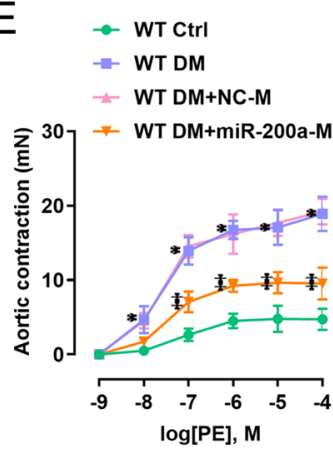

$\mathrm{F}$
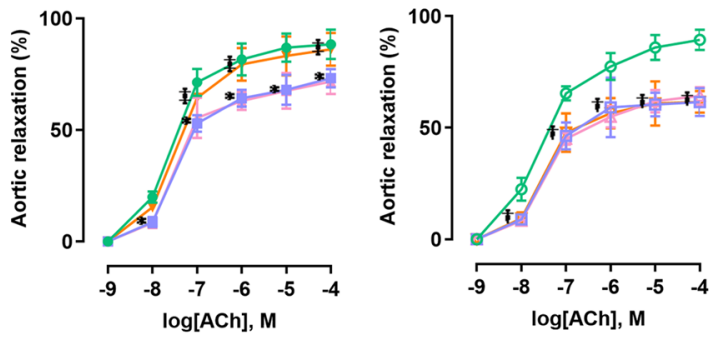

found under the conditions of hyperglycemia/HG, and contributed to endothelial injuries, such as ED, oxidative damage and inflammation. These findings urge more potent and alternative approaches to enhance the NRF2 antioxidant capacity in protection against diabetic ED.

In recent years, attempts have been made to activate NRF2 in the endothelium. Baicalin, a functional component in scutellaria baicalensis root, ameliorated HG-induced endothelial OS and inflammation through AKT/GSK-3ק/Fyn-mediated NRF2 activation (Chen et al. 2018). Resveratrol activated NRF2, attenuated HG-induced OS and inflammation in coronary arterial endothelial cells, and improved ED in high-fat diet-fed mice (Ungvari et al. 2010). Recently, we reported that sodium butyrate - a fatty acid derivative found in human diet and

\section{Figure 6}

miR-200a-M improved hyperglycemia-induced aortic OS, inflammation and ED through activation of NRF2. To assess OS in the aortas of the WT and Nrf2 KO mice, (A) iNos mRNA levels were measured by $q P C R$, and (B) 4-HNE were detected by $\mathrm{IHC}$ staining, with positively stained area quantified. To evaluate aortic inflammation, (C) Vcam-1 and (D) Mcp-1 mRNA levels were determined by qPCR. To analyze $E D$, vascular $(E)$ contraction in response to $\mathrm{PE}$, and $(\mathrm{F})$ relaxation in response to $\mathrm{ACh}$ were determined using aortic rings from the WT and Nrf2 KO mice. For (A-D), the data were normalized to WT Ctrl. All the data were presented as means \pm s.D. $(n=7) .{ }^{*} P<0.05$ vs WT Ctrl. ${ }^{\dagger} P<0.05$ vs KO Ctrl. $¥ P<0.05$ vs WT DM + NC-M. 4-HNE, 4-hydroxynonenal; ACh, acetylcholine; ED, endothelial dysfunction; iNOS, inducible nitric oxide synthase; $\mathrm{PE}$, phenylephrine. Other abbreviations are the same as Figs 1, 2, 4 and 5. also an inhibitor of histone deacetylase - improved diabetic ED through P300-mediated transcriptional activation of the Nrf2 gene (Wu et al. 2018b). As KEAP1 is a key negative regulator of NRF2, in a previous report, activation of NRF2 in the diabetic endothelium was achieved by dh404 - a small molecule inhibitor of KEAP1 (Sharma et al. 2017). Decreasing Keap1 mRNA levels or hampering the translation process of the KEAP1 protein (as opposed to the structural inactivation of KEAP1) may provide an alternative and viable approach for activating NRF2. Our findings support this notion, showing that miR-200a activated NRF2 through degradation of Keap1 mRNA (Figs 2B, C, D, E, F, 3A, B, C, D, E, F and 5C, D, E, F, G, H, I, J).

In the present study, miR-200a levels were decreased under $\mathrm{HG}$ in a time-dependent manner (Fig. 1A). 
In accordance with this observation, a remarkably lower miR-200a level was found in the aortas of the diabetic mice compared with the non-diabetic mice (Fig. 5C). To date, it is still unclear how HG or hyperglycemia reduces miR-200a levels in ECs or the endothelium. Aldose reductase was previously reported to be increased in the diabetic kidney, and accounts for the decrease in miR-200a level, providing a clue for the regulation of miR-200a in the endothelium under hyperglycemic conditions (Wei et al. 2014). This speculation needs to be investigated in future work.

Specific antagomirs are chemically engineered oligonucleotides that prevent other molecules from binding to a desired miRNA. In this regard, an antagomir is supposed to form a dimer with the target miRNA, blocking the target miRNA's ability to bind its target mRNA. However, antagomirs often led to decrease in the levels of target miRNAs, as shown in the present study (Fig. 2A) and many others (Putta et al. 2012, Wei et al. 2014, Wu et al. 2016). This might be caused by bindinginduced instability of the target miRNAs, the effect of which should contribute to the inhibition of the target miRNA's efficacies.

A notable difference in the expression pattern was found between endothelial t-NRF2 and n-NRF2 under the time-course incubation with HG (Fig. 1D and E). Particularly, HG time-dependently decreased t-NRF2 levels, whereas HG increased n-NRF2 firstly and lowered n-NRF2 thereafter (Fig. 1D and E). This effect could be explained by the expression of KEAP1 protein under HG. KEAP1 is known to induce degradation of NRF2 via proteasome. As KEAP1 protein accumulated under HG (Fig. 1C), t-NRF2 level probably declined through degradation (Fig. 1D). n-NRF2 - a portion of t-NRF2 - translocated into the nuclei in response to HG for 6 and 12 hours (Fig. 1E), serving as a protective compensatory response to the stimulus HG. However, when KEAP1 was dramatically increased under HG for 24 and 48 hours (Fig. 1C), it significantly restricted NRF2 from nuclear translocation, resulting in less n-NRF2 (Fig. 1E). As a consequence of the decrease in n-NRF2 levels at $48 \mathrm{~h}$, the mRNA levels of Nqo1 and Ho1 were drastically decreased (Fig. 1F). This had resulted in a significant elevation of OS and inflammation at $48 \mathrm{~h}$ (Fig. 1G), as a decompensatory stage during a longer period of stimulation with HG. In accordance with this decompensatory effect in vitro, our in vivo study has found that aortic NRF2 antioxidant signaling was blunted under long-term hyperglycemic conditions for 20 weeks (Fig. 5G, H, I and J). The remaining level of NRF2 antioxidant signaling under hyperglycemic conditions
(Fig. 5G, H, I and J), although basal, still offered protection, since hyperglycemia resulted in more severe aortic OS and inflammation in the Nrf2 $\mathrm{KO}$ mice compared with the WT mice (Fig. 6A, B, C and D).

In previous studies, DM-induced OS and inflammation were evident in both the intima and adventitia (Tan et al. 2014, Wu et al. 2018a,b, 2019), where in the present study 4 -HNE was found to be predominantly expressed (Fig. $6 \mathrm{~B})$. The enhanced OS and inflammation in the adventitia might be caused by adventitial ECs generated from neovascularization (O'Brien et al. 1993), and adventitial fibroblasts that is known to produce substantial amounts of ROS in response to vascular injury (Csanyi et al. 2009). It would be interesting to study the role of the adventitia in diabetic macrovascular diseases in future studies.

NRF2 has proven to be a viable drug target as evidenced by the use of dimethyl fumarate (also known as BG-12) - an inhibitor of KEAP1 - for the treatment of multiple sclerosis (Gold et al. 2012). More recently, sulforaphane - another inhibitor of KEAP1 - was shown in a pilot clinical trial to prevent carcinogen-induced oral cancer by activating NRF2 antioxidant signaling (Bauman et al. 2016). To date, activation of NRF2 has not been investigated in clinical trials for diabetic ED. The present study may provide an experimental basis for future clinical trials for diabetic ED. Notably, different from structural inhibition of the KEAP1 protein, targeting Keap1 mRNA and its upstream miR-200a has shown novel strategies toward the activation of NRF2 in the endothelium.

Taken together, the present study has found that miR-200a improved diabetic ED via modulating KEAP1/NRF2 antioxidant signaling, demonstrating a critical role of NRF2 in protection against diabetic ED, and providing miR-200a and Keap1 mRNA as potential targets in future management of diabetic macrovascular complications.

\section{Declaration of interest}

The authors declare that there is no conflict of interest that could be perceived as prejudicing the impartiality of the research reported.

\section{Funding}

This work was supported in part by the National Natural Science Foundation of China $(81973031,81600573)$ and Cheeloo Young Scholar Program of Shandong University (21320089963054) to Hao Wu; Natural Science Foundation of Jilin Province (2018SCZWSZX-045) to Ziping Jiang; Key Technology Research and Development Program of Shandong Province (2019GSF108147) to Jun Jiang; National Natural Science Foundation of China (81901106) and Youth Talent Found of The Second Hospital of Shandong University (2018YT09) to Linlin Xu; China Postdoctoral Science Foundation (2018M632686), Young Scholars Program of Shandong 
University (2018WLJH34) and Fundamental Research Funds of Shandong University (2017TB0029) to Lei Du; National Natural Science Foundation of China (81774393) to Zhaohui Wang; and National Natural Science Foundation of China (81771329) to Laijin Lu.

\section{Author contribution statement}

Hao Wu conceived and designed the project. Ziping Jiang, Junduo Wu, Fuzhe Ma, Jun Jiang, Linlin Xu, Lei Du, Wenlin Huang, Zhaohui Wang, Ye Jia, Laijin Lu and Hao Wu researched/interpreted the data. Hao Wu, Ziping Jiang and Laijin Lu wrote the manuscript. Hao Wu, Laijin Lu, Ziping Jiang, Junduo Wu, Fuzhe Ma, Jun Jiang, Linlin Xu, Lei Du, Wenlin Huang, Zhaohui Wang and Ye Jia discussed, reviewed and revised the manuscript. Hao Wu, Ziping Jiang, Jun Jiang, Linlin $\mathrm{Xu}$, Lei Du, Zhaohui Wang and Laijin Lu provided funding. All the authors approve the version to be published.

\section{References}

Barwari T, Eminaga S, Mayr U, Lu R, Armstrong PC, Chan MV, Sahraei M, Fernandez-Fuertes M, Moreau T, Barallobre-Barreiro J, et al. 2018 Inhibition of profibrotic microRNA-21 affects platelets and their releasate. JCI Insight 3 123335. (https://doi.org/10.1172/jci. insight.123335)

Bauman JE, Zang Y, Sen M, Li C, Wang L, Egner PA, Fahey JW, Normolle DP, Grandis JR, Kensler TW, et al. 2016 Prevention of carcinogen-induced oral cancer by sulforaphane. Cancer Prevention Research 9 547-557. (https://doi.org/10.1158/1940-6207.CAPR-15-0290)

Betteridge DJ 2000 What is oxidative stress? Metabolism: Clinical and Experimental 49 (Supplement 1) 3-8. (https://doi.org/10.1016/s00260495(00)80077-3)

Ceriello A, Novials A, Ortega E, Canivell S, La Sala L, Pujadas G, Esposito K, Giugliano D \& Genovese S 2013 Glucagon-like peptide 1 reduces endothelial dysfunction, inflammation, and oxidative stress induced by both hyperglycemia and hypoglycemia in type 1 diabetes. Diabetes Care 36 2346-2350. (https://doi.org/10.2337/dc12-2469)

Chen G, Chen X, Niu C, Huang X, An N, Sun J, Huang S, Ye W, Li S, Shen Y, et al. 2018 Baicalin alleviates hyperglycemia-induced endothelial impairment 1 via Nrf2. Journal of Endocrinology $\mathbf{2 4 0}$ 81-98. (https://doi.org/10.1530/JOE-18-0457)

Csanyi G, Taylor WR \& Pagano PJ 2009 NOX and inflammation in the vascular adventitia. Free Radical Biology and Medicine 47 1254-1266. (https://doi.org/10.1016/j.freeradbiomed.2009.07.022)

Deshpande SD, Putta S, Wang M, Lai JY, Bitzer M, Nelson RG, Lanting LL, Kato M \& Natarajan R 2013 Transforming growth factor-betainduced cross talk between p53 and a microRNA in the pathogenesis of diabetic nephropathy. Diabetes 62 3151-3162. (https://doi. org/10.2337/db13-0305)

Eades G, Yang M, Yao Y, Zhang Y \& Zhou Q 2011 miR-200a regulates Nrf2 activation by targeting Keap1 mRNA in breast cancer cells. Journal of Biological Chemistry 286 40725-40733. (https://doi.org/10.1074/jbc. M111.275495)

Fabian MR, Sonenberg N \& Filipowicz W 2010 Regulation of mRNA translation and stability by microRNAs. Annual Review of Biochemistry 79 351-379. (https://doi.org/10.1146/annurev-biochem-060308-103103)

Forbes JM, Yee LT, Thallas V, Lassila M, Candido R, Jandeleit-Dahm KA, Thomas MC, Burns WC, Deemer EK, Thorpe SR, et al. 2004 Advanced glycation end product interventions reduce diabetes-accelerated atherosclerosis. Diabetes 53 1813-1823. (https://doi.org/10.2337/ diabetes.53.7.1813)

Giacco F \& Brownlee M 2010 Oxidative stress and diabetic complications. Circulation Research 107 1058-1070. (https://doi.org/10.1161/ CIRCRESAHA.110.223545)
Gold R, Kappos L, Arnold DL, Bar-Or A, Giovannoni G, Selmaj K, Tornatore C, Sweetser MT, Yang M, Sheikh SI, et al. 2012 Placebocontrolled phase 3 study of oral BG-12 for relapsing multiple sclerosis. New England Journal of Medicine 367 1098-1107. (https://doi. org/10.1056/NEJMoa1114287)

Incalza MA, D'Oria R, Natalicchio A, Perrini S, Laviola L \& Giorgino F 2018 Oxidative stress and reactive oxygen species in endothelial dysfunction associated with cardiovascular and metabolic diseases. Vascular Pharmacology 100 1-19. (https://doi.org/10.1016/j. vph.2017.05.005)

Kaspar JW, Niture SK \& Jaiswal AK 2009 Nrf2:INrf2 (Keap1) signaling in oxidative stress. Free Radical Biology and Medicine 47 1304-1309. (https://doi.org/10.1016/j.freeradbiomed.2009.07.035)

Kato M, Wang M, Chen Z, Bhatt K, Oh HJ, Lanting L, Deshpande S, Jia Y, Lai JY, O'Connor CL, et al. 2016 An endoplasmic reticulum stressregulated IncRNA hosting a microRNA megacluster induces early features of diabetic nephropathy. Nature Communications 712864 . (https://doi.org/10.1038/ncomms12864)

Kim JH, Choi YK, Lee KS, Cho DH, Baek YY, Lee DK, Ha KS, Choe J, Won MH, Jeoung D, et al. 2012 Functional dissection of Nrf2dependent phase II genes in vascular inflammation and endotoxic injury using Keap1 siRNA. Free Radical Biology and Medicine $\mathbf{5 3}$ 629-640. (https://doi.org/10.1016/j.freeradbiomed.2012.04.019)

Laight DW, Carrier MJ \& Anggard EE 2000 Antioxidants, diabetes and endothelial dysfunction. Cardiovascular Research 47 457-464. (https:// doi.org/10.1016/s0008-6363(00)00054-7)

O'Brien KD, Allen MD, McDonald TO, Chait A, Harlan JM, Fishbein D, McCarty J, Ferguson M, Hudkins K \& Benjamin CD 1993 Vascular cell adhesion molecule- 1 is expressed in human coronary atherosclerotic plaques. Implications for the mode of progression of advanced coronary atherosclerosis. Journal of Clinical Investigation 92 945-951. (https://doi.org/10.1172/JCI116670)

Pan C, Zhou S, Wu J, Liu L, Song Y, Li T, Ha L, Liu X, Wang F, Tian J, et al. 2017 NRF2 plays a critical role in both self and EGCG protection against diabetic testicular damage. Oxidative Medicine and Cellular Longevity 2017 3172692. (https://doi.org/10.1155/2017/3172692)

Peng J, Li X, Zhang D, Chen JK, Su Y, Smith SB \& Dong Z 2015 Hyperglycemia, p53, and mitochondrial pathway of apoptosis are involved in the susceptibility of diabetic models to ischemic acute kidney injury. Kidney International 87 137-150. (https://doi. org/10.1038/ki.2014.226)

Putta S, Lanting L, Sun G, Lawson G, Kato M \& Natarajan R 2012 Inhibiting microRNA-192 ameliorates renal fibrosis in diabetic nephropathy. Journal of the American Society of Nephrology 23 458-469. (https://doi.org/10.1681/ASN.2011050485)

Ruiz S, Pergola PE, Zager RA \& Vaziri ND 2013 Targeting the transcription factor Nrf2 to ameliorate oxidative stress and inflammation in chronic kidney disease. Kidney International 83 1029-1041. (https:// doi.org/10.1038/ki.2012.439)

Sena CM, Pereira AM \& Seica R 2013 Endothelial dysfunction - a major mediator of diabetic vascular disease. Biochimica et Biophysica Acta 1832 2216-2231. (https://doi.org/10.1016/j.bbadis.2013.08.006)

Sharma A, Rizky L, Stefanovic N, Tate M, Ritchie RH, Ward KW \& de Haan JB 2017 The nuclear factor (erythroid-derived 2)-like 2 (Nrf2) activator dh404 protects against diabetes-induced endothelial dysfunction. Cardiovascular Diabetology 16 33. (https://doi. org/10.1186/s12933-017-0513-y)

Sun W, Liu X, Zhang H, Song Y, Li T, Liu X, Liu Y, Guo L, Wang F, Yang T, et al. 2017 Epigallocatechin gallate upregulates NRF2 to prevent diabetic nephropathy via disabling KEAP1. Free Radical Biology and Medicine 108 840-857. (https://doi.org/10.1016/j. freeradbiomed.2017.04.365)

Tan SM, Sharma A, Stefanovic N, Yuen DY, Karagiannis TC, Meyer C, Ward KW, Cooper ME \& de Haan JB 2014 Derivative of bardoxolone methyl, dh404, in an inverse dose-dependent manner lessens https://joe.bioscientifica.com https://doi.org/10.1530/JOE-19-0414 (c) 2020 Society for Endocrinology Published by Bioscientifica Ltd. Printed in Great Britain 
diabetes-associated atherosclerosis and improves diabetic kidney disease. Diabetes 63 3091-3103. (https://doi.org/10.2337/db13-1743)

Ungvari Z, Bagi Z, Feher A, Recchia FA, Sonntag WE, Pearson K, de Cabo R \& Csiszar A 2010 Resveratrol confers endothelial protection via activation of the antioxidant transcription factor Nrf2. American Journal of Physiology: Heart and Circulatory Physiology 299 H18-H24. (https://doi.org/10.1152/ajpheart.00260.2010)

Wang HJ, Huang YL, Shih YY, Wu HY, Peng CT \& Lo WY 2014 MicroRNA146a decreases high glucose/thrombin-induced endothelial inflammation by inhibiting NAPDH oxidase 4 expression. Mediators of Inflammation 2014 379537. (https://doi.org/10.1155/2014/379537)

Wei J, Zhang Y, Luo Y, Wang Z, Bi S, Song D, Dai Y, Wang T, Qiu L, Wen L, et al. 2014 Aldose reductase regulates miR-200a-3p/141-3p to coordinate Keap1-Nrf2, Tgfbeta1/2, and Zeb1/2 signaling in renal mesangial cells and the renal cortex of diabetic mice. Free Radical Biology and Medicine 67 91-102. (https://doi.org/10.1016/j. freeradbiomed.2013.10.811)

Wu H, Zhou S, Kong L, Chen J, Feng W, Cai J, Miao L \& Tan Y 2015 Metallothionein deletion exacerbates intermittent hypoxia-induced renal injury in mice. Toxicology Letters 232 340-348. (https://doi. org/10.1016/j.toxlet.2014.11.015)

Wu H, Kong L, Tan Y, Epstein PN, Zeng J, Gu J, Liang G, Kong M, Chen X, Miao L, et al. 2016 C66 ameliorates diabetic nephropathy in mice by both upregulating NRF2 function via increase in miR-200a and inhibiting miR-21. Diabetologia 59 1558-1568. (https://doi. org/10.1007/s00125-016-3958-8)

Wu H, Wu J, Zhou S, Huang W, Li Y, Zhang H, Wang J \& Jia Y $2018 a$ SRT2104 attenuates diabetes-induced aortic endothelial dysfunction via inhibition of P53. Journal of Endocrinology 237 1-14. (https://doi. org/10.1530/JOE-17-0672)

Wu J, Jiang Z, Zhang H, Liang W, Huang W, Zhang H, Li Y, Wang Z, Wang J, Jia Y, et al. 2018b Sodium butyrate attenuates diabetes- induced aortic endothelial dysfunction via P300-mediated transcriptional activation of Nrf2. Free Radical Biology and Medicine 124 454-465. (https://doi.org/10.1016/j.freeradbiomed.2018.06.034)

Wu J, Liang W, Tian Y, Ma F, Huang W, Jia Y, Jiang Z \& Wu H 2019 Inhibition of P53/miR-34a improves diabetic endothelial dysfunction via activation of SIRT1. Journal of Cellular and Molecular Medicine 23 3538-3548. (https://doi.org/10.1111/jcmm.14253)

Yang T, Peleli M, Zollbrecht C, Giulietti A, Terrando N, Lundberg JO, Weitzberg E \& Carlstrom M 2015 Inorganic nitrite attenuates NADPH oxidase-derived superoxide generation in activated macrophages via a nitric oxide-dependent mechanism. Free Radical Biology and Medicine 83 159-166. (https://doi.org/10.1016/j. freeradbiomed.2015.02.016)

Yaribeygi H, Mohammadi MT, Rezaee R \& Sahebkar A 2018 Crocin improves renal function by declining Nox-4, IL-18, and p53 expression levels in an experimental model of diabetic nephropathy. Journal of Cellular Biochemistry 119 6080-6093. (https://doi. org/10.1002/jcb.26806)

Zhang DD \& Hannink M 2003 Distinct cysteine residues in Keap1 are required for Keap1-dependent ubiquitination of Nrf2 and for stabilization of Nrf2 by chemopreventive agents and oxidative stress. Molecular and Cellular Biology 23 8137-8151. (https://doi.org/10.1128/ mcb.23.22.8137-8151.2003)

Zhao Q, Zhang F, Yu Z, Guo S, Liu N, Jiang Y, Lo EH, Xu Y \& Wang X 2019 HDAC3 inhibition prevents blood-brain barrier permeability through Nrf2 activation in type 2 diabetes male mice. Journal of Neuroinflammation 16 103. (https://doi.org/10.1186/s12974-019. 1495-3)

Zheng H, Whitman SA, Wu W, Wondrak GT, Wong PK, Fang D \& Zhang DD 2011 Therapeutic potential of Nrf2 activators in streptozotocin-induced diabetic nephropathy. Diabetes $603055-3066$. (https://doi.org/10.2337/db11-0807)

Received in final form 19 December 2019

Accepted 7 February 2020

Accepted Manuscript published online 7 February 2020 (c) 2020 Society for Endocrinology Published by Bioscientifica Ltd. Printed in Great Britain 